QK329

.M6

Morton, Friedrich

Beiträge zur kenntnis der flora von Nord-Dalmatien 


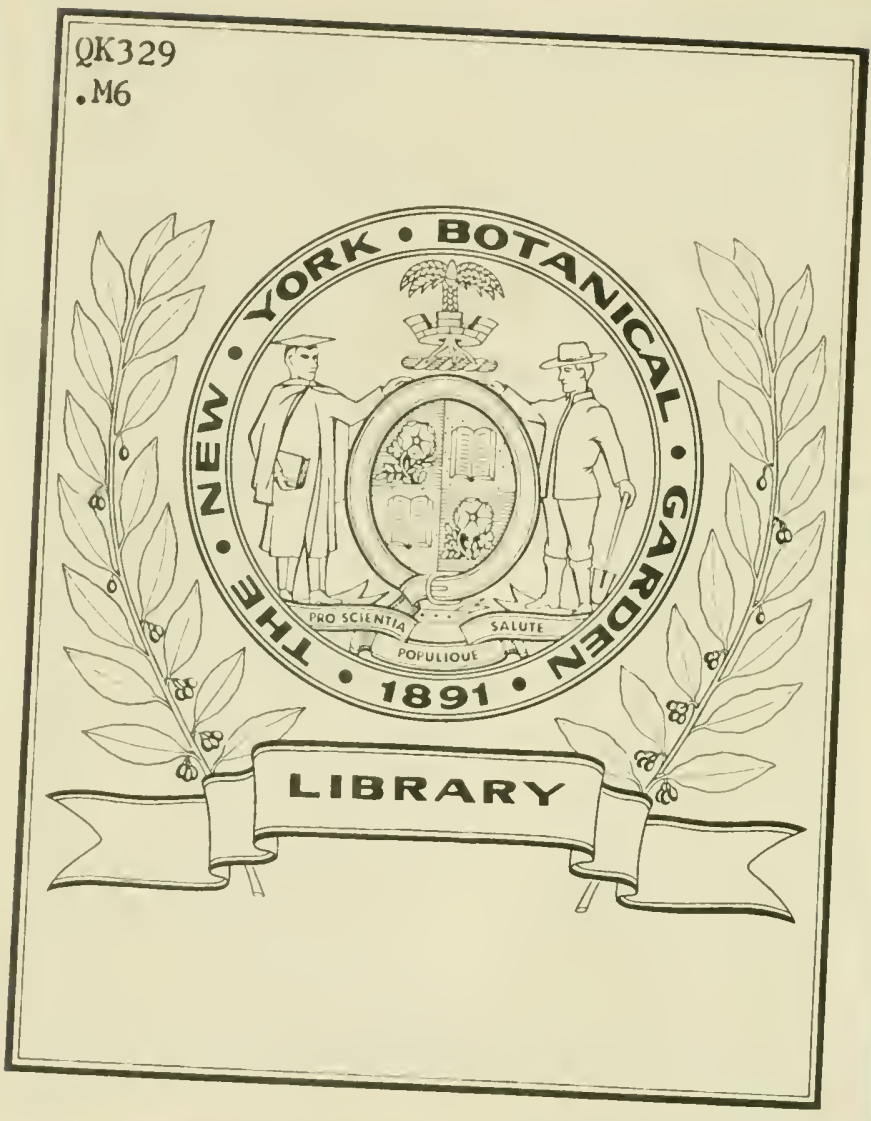






\title{
Beiträge zur Kenntnis der Flora von Nord-Dalmatien.
}

\author{
Von Friedrich Morton (Wien).
}

(Mit 4 Textabbildungen.)

Aus der Summe der von mir gelegentlich der pflanzengeographischen Durchforschung der Insel Arbe und deren Nachbarinseln gemachten Pflanzenfunde veröffentliche ich im folgenden einige, die mir aus irgend einem Grunde von Interesse $\mathrm{zu}$ sein scheinen ${ }^{1}$ ).

Polypodium vulgare L. var. serratum Willd. f. triangulare Hausm. ${ }^{2}$ ). Insel Arbe: auf einem Kalkfelsen am $\mathrm{N}$-Absturze des Cruna vrh.

Phyllitis hemionitis (Lag.) 0. Kuntze. Insel Arbe: in der Formation der Felsflur am NO-Absturze des Tignarogebirges.

Ficus carica L. erinosyce Tschirch et Rarasini. Inseln Arbe und S. Gregorio: als Felsenpflanze am NO-Absturze beider Inseln.

Polygonum maritimum L. Insel Arbe: auf feinem Meeresschotter in der Valle S. Cristoforo; tonangebende Leitpflanze auf den Sanddünen am SO-Ende des Loparotales.

$P$. aviculare I. var. litorale Koch. Insel Arbe: in der Formation des schotterigen Meeresstrandes bei der Stadt Arbo und in der Formation der Salztriften am SO-Ende der Campora.

Euphorbia literata Jacq. Insel Arbe: an salzhaltigen Stellen im Paludo und am SO-Ende der Campora.

E. pinea L. Insel Arbe: in der Formation des schotterigen Meeresstrandes in der $\nabla$. S. Cristoforo und in der Formation der Salztriften am SOEnde der Campora.

Theligonum cynocrambe L. Insel Dolin: am SW-Abhange der Insel (K.). Sagina maritima Don. Insel Arbe: an einem Wege beim Kloster S. Eufemia: Insel S. Gregorio: in der Formation der Strandklippen bei der Pta. Plitvac.

Dianthus ciliatus Guss. Die Art zerfällt in drei von Visiani (Flora dalmat., III, 162) gut unterschiedene. Varietäten; die Pflanzen des Gebietes stimmen mit den Originalen des Herbars $\mathrm{V}$ isiani vollkommen überein ${ }^{3}$ ). Ich gebe im folgenden die Diagnosen der drei Varietäten, die ich gegenüber den von $V$ isiani aufgestellten nach Durchsicht eines reichen Materials etwas geändert und erweitert habe.

1) Es wurden hier auch einzelne Funde des Herrn Dr. B. Kü mmerle bèrücksichtigt, der mir für meine Monographie in liebenswürdiger Weise das gesamte ron ihm auf Arbe gesammelte Material zur Verfügung gestellt hatte, wofür ich ihm auch an dieser Stelle herzlichst danke. In den betreffenden Fällen ist sein Name (K.) dem Standorte beigefügt.

2) Revid. Paulin.

3) Herr Prof. A. Béguinot (Padua) hatte die Liebenswürdigkeit, mir die Visianischen Originale zu senden, wofür ich ihm auch an dieser Stelle bestens danke. 
var. a raccmosus (Abb. 1): grundständige Blätter bis $10 \mathrm{~cm}$ lang, Stêngel bis oben beblättert, Stengelblättel $2-5 \mathrm{~cm}$ lang. Blüten sitzend oder fast sitzend, oder die unteren \pm gestielt und nur einzeln, die oberen aber meist zu 2-5büschelig und fast traubig angeordnet. Blumenblätter elliptisch bis lanzettlich.

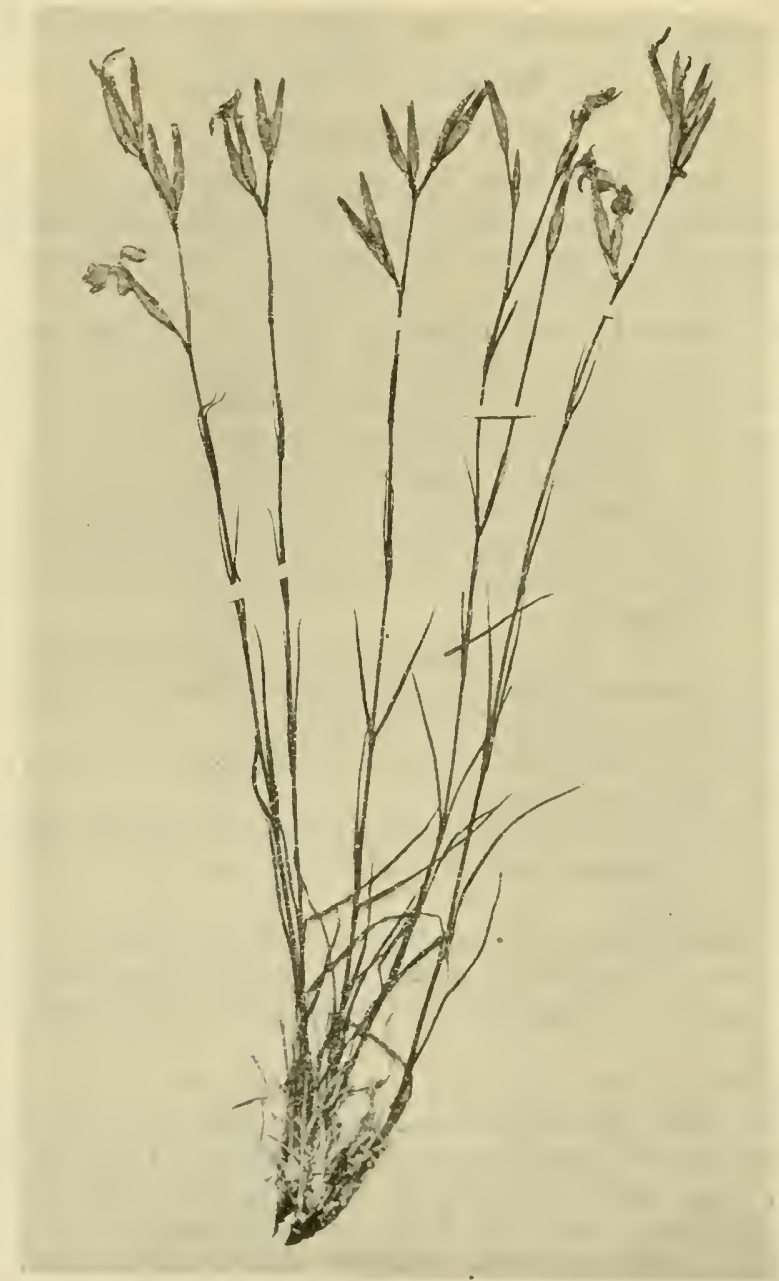

Abb. 1 Dianthus ciliatus Guss. var. $\alpha$ racemosus Vis.

var. $\beta$ cymosus (Abb. $2 \mathrm{a})$ : Blätter kürzer und steifer, Stengelblätter meist nur $1-3 \mathrm{~cm}$ lang. Blüten lang (bis $7 \mathrm{~cm}$ ), gestielt, fast immer einzeln, rispig angeordnet. Blumenblätter eiförmig.

var. $\gamma$ Brocchianus (Abb. 2b): Blätter sehr kurz, die grundständigen mit kurzer Ḱnorpelspitze, eine Rosette aus meist nur $1(-2)$ 
Zentimeter langen, sehr starren und spitzen Blättern bildend. Stengelblätter meist stark reduziert. Meist nur 1-3 Stengel, diese ein-, selten zweiblütig, manchmal nur bis $2 \mathrm{~cm}$ hoch. Eine sehr charakteristische Form.

a) Iısel Arbe: in der Garrigue auf der Landzunge Fercagno.

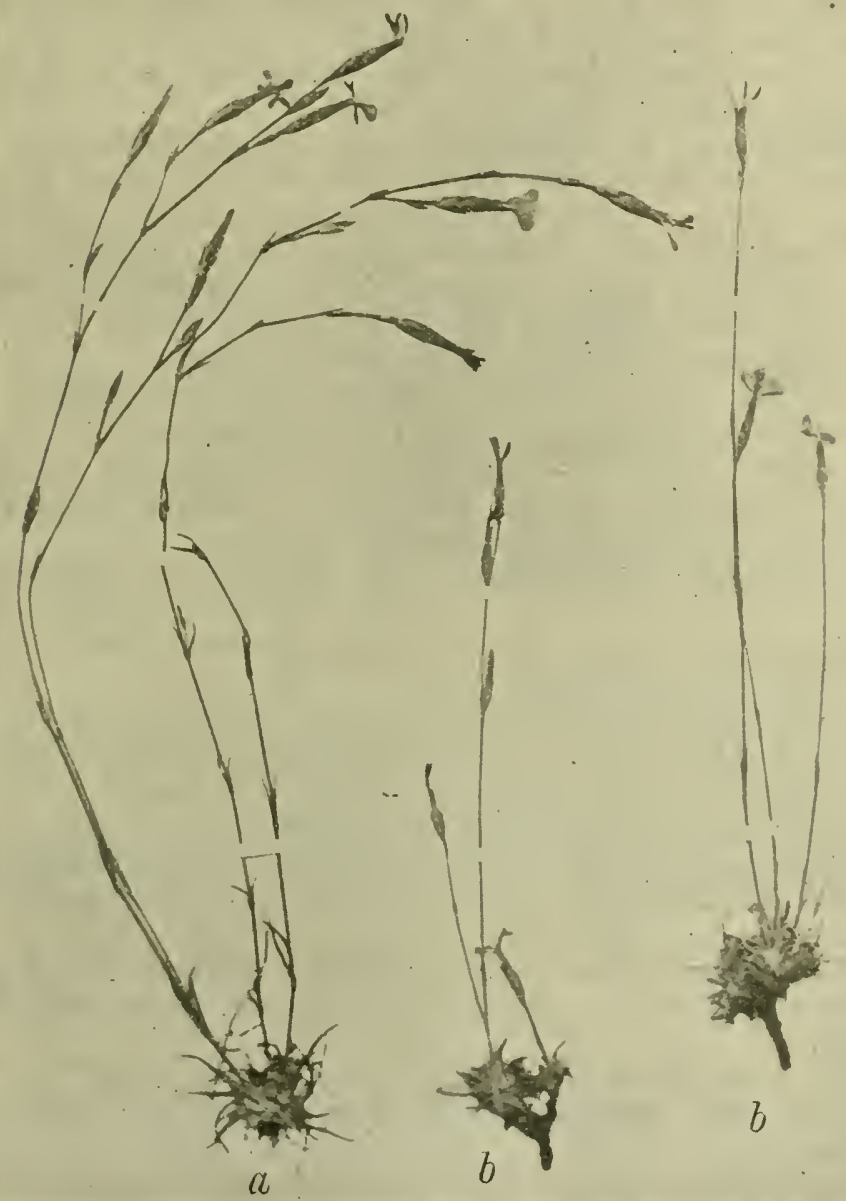

Abb. 2. Dianthus ciliatus Guss. var. $\beta$ cymosus Vis. $(2 a)$ und var. $\gamma$ Brocchiauus Vis. $(2 b)$.

$\beta)$ Insel Arbe: in der Garrigue am NO-Absturze des Cruna vrh und in der Formation der Felsflur am NO-Absturze des Tignarogebirges.

$\gamma)$ Insel Arbe: in der Garrigue im südlichen Teile der Halbinsel Loparo. 
Delphinium staphisagria L. Insel Goli: auf einem feuchten Schuttabhang bei "Domolo".

Rununculus ophioglossifolius Vill. var. fontanus Presl f. natans Glück ${ }^{1}$ ). Insel Arbe: in Entwässerungsgräben in der Campora. In dieser Form bisher nur aus Sizilien bekannt.

Fumaria parviflora Lam. Insel Arbe: auf einem Acker bei Barbato (Rossi).

F. capreolata L. Insel Arbe: in einer Paliurus-Hecke beim Kloster S. Eufemia und an einer Mauer bei Barbato.

Alyssum Arduini Fritsch. Inseln S. Gregorio und Goli: in der Formation der Felsflur am NO-Absturze beider Inselu.

Peltaria alliacea Jacq. f. crassifolia mihi. An schattig feuchten Felsen in der Formation der Felsflur am NO.Absturze der Inseln Arbe und S. Gregorio. Diese durch die eigentümlichen Standortsverhältnisse (große Feuchtigkeit und starken Schatten, starken Salzgehalt der Luft und des Bodens bei Bora) bedingte Form unterscheidet sich rom Typus durch ihren üppigen, bis $50 \mathrm{~cm}$ hohen Wuchs und große, ziemlich dicke und fleischige, bläulich bereifte Blätter.

Tuberaria guttata (L.) Fourreau f. vulgaris (Willk.) Janchen. Insel Arbe: auf Weideboden in der „Fruga“-Alluvialmulde.

Fumana vulgaris Spach. ${ }^{2}$ ). Insel Arbe: in der Garrigue am NO-Absturze des Cruna vrh und auf der Halbinsel Loparo sowie auf den Sanddünen am SO-Ende des Loparotales.

Ruta divaricata Ten. Insel Dolin: in der Garrigue im mittleren Teile der Insel.

Cotinus coggygria Scop. Insel Arbe: in der Formation der Felsflur am NOAbsturze des 'Tignarogebirges.

Rhamnus intermedia Steud. et Hochst. Insel Arbe: in der steinigen Trift am SW-Abhange (K.) und NO-Absturze des lignarogebirges; Insel S. Gregorio: in der steinigen Trift am NO-Absturze.

Rh. rupestris Scop. Insel Arbe: am NO-Absturze des Tignarogebirges. Sedum dasyphyllum L. Insel Arbe: zerstreut in der steinigen Trift auf dem ganzen Tignarogebirge (auch K.); Insel Dolin: in der steinigen Trift (K.).

Rosa dumetorum Thuill. var. affinita Thuill. subvar. Euphemiae H. Braun nova ${ }^{3}$ ) subvar. Insel Arbe: in einer Hecke beim Kloster S. Eufemia.

Foliis majoribus et magis rotundatis quam in typo.

R. agrestis Savi var. Mortonii H. Braun nova var. Insel Arbe: im Dundowalde.

Rami aculeati aculeis aduncis hinc inde validis et subverticillatis. Stipulae glandulosae angustatae. Petioli aculeolati, glandulosi et hinc inde pilis singulis obtecti. Folia parva vel mediocria ad basin attenuata hinc inde subcuneata supra glabra vel leviter pilosula, subtus dense glandulis obtecta in margine argute multiserrata ut in Sepiaceis. Bracteae glabrae, in dorso ad nervum medium glandulosae, in marginibus dense glandulis praeditae. Pedunculi glabri non elongati.

1) Det. H. Glü ck.

2) Revid. E. Jan chen.

3) Alle Rosen von H. Braun bestiment. 
Receptacula ovoidea vel breviter ellipsoidea. Styli abbreviati, pilosi. Petala pallide rosacea, parva. Sepala in dorso pilosa, in margine glandulis validis obtecta post anthesin reflexa. Differt a typo stylis pilosis non elevatis, a $R$. virgultorum Rip. receptaculis non globosis sepalis non glandulosis valide obtectis etc. A Rosa inodora Fries differt aculeis non falcatis, sepalis post anthesin distantibus.

Pyracantha coccinea Roem.'). Insel Arbe: iu der steinigen Trift am NOAbsturze des Tignarogebirges.

Astragalus Muelleri Steud. et Hochstett. Inseln Dolin und Goli: sehr vereinzelt in der steinigen Trift beider Inselı.

Medicago marina L. Insel Arbe: am Meeresstrand bei der Stadt Arbe; tonangebende Leitpflanze auf den Sanddünen am SO-Ende des Loparotales.

Trifolium rubens L. Insel Arbe: Bestand von Quercus ilex und Q. lanuginosa westlich des Paludo.

T. lappaceım L. Insel Arbe: steinige Ufer beim Paludo (K.).

T. pallidum W. K. Insel Arbe: im Dundowalde.

Bunium divaricatum Bert. Inseln Arbe, Dolin und Goli: in der steinigen 'Trift auf den drei Inseln.

Seseli tortuosum L. Insel Arbe: in der Garrigue bei der Pta. Stolac (Halbinsel Loparo).

Libunotis daucifolia (Scop.) Rebb. ${ }^{2}$ ). Inseln Arbe und S. Gregorio: in der Formation der Felsflur am NO-Absturze beider Inseln.

Torilis nodosa (L.) Gaertn. Insel Dolin: in der Garrigue im nördlichen Teile der Insel.

Statice virgata Willd. Insel Arbe: in der Formation der Strandklippen im westlichen Teile der Insel und auf dem Scoglio Zrinja.

Convolvulus arvensis L. var. lancifolius Presl ${ }^{3}$ ) (Abb. 3). Insel Arbe: auf einem Brachacker bei der Stadt Arbe. Ich beobachtete diese Varietät durch mebrere Jahre am selben Standort. Die Länge der Blätter beträgt im Durchschnitt $6 \mathrm{~cm}$, die Breite $0 . \overline{\mathrm{cm}}$.

Calystegia soldanella (L.) R. Br. Insel Arbe: tonangebende Leitpflanze auf dem Saudstrande am SO-Ende des Loparotales.

Echium plantagineum L. Insel Arbe: Ruderalplätze beim „Campo Marzio".

E. parviflorum Mnch. Insel Arbe: ebenda.

Melampyrum versicolor (Posp.) Fritsch ${ }^{4}$ ). Insel Arbe: im Bestande ron Quercus lanuginosa in der. Mundanje glava.

Utricularia neglecta Lehm. ${ }^{5}$ ). Insel Arbe: in einem Brackwassergraben am SO-Ende des Loparotales. Neu für Dalmatien.

Stachys muritima L. Insel Arbe: auf den Sanddünen am SO-Ende des Loparotales.

1) Diese Pflanze wurde auberdem von A. Ginzberger in Dalmation an folgenden Orten gefunden: In einer Föhrenaufforstung bei der Eisenbahnstation PerkovicSlivno; am Wege ron Scardona zu den Kerkafällen; auf dem Scoglio Planchetta.

2) Revid. A. v. Hayek.

3) Revid. A Béguinot.

4) Det. H. Poeverlein.

5) Revid. H. Glüek. Dieser Fund wurde mit meinem Einverständnis bereits von $\mathrm{Poeverlein} \mathrm{in} \mathrm{Kneuckers} \mathrm{Allgem.} \mathrm{botan.} \mathrm{Zeitschr.,} \mathrm{Jahrg.} \mathrm{1913,} \mathrm{publiziert.}$ 


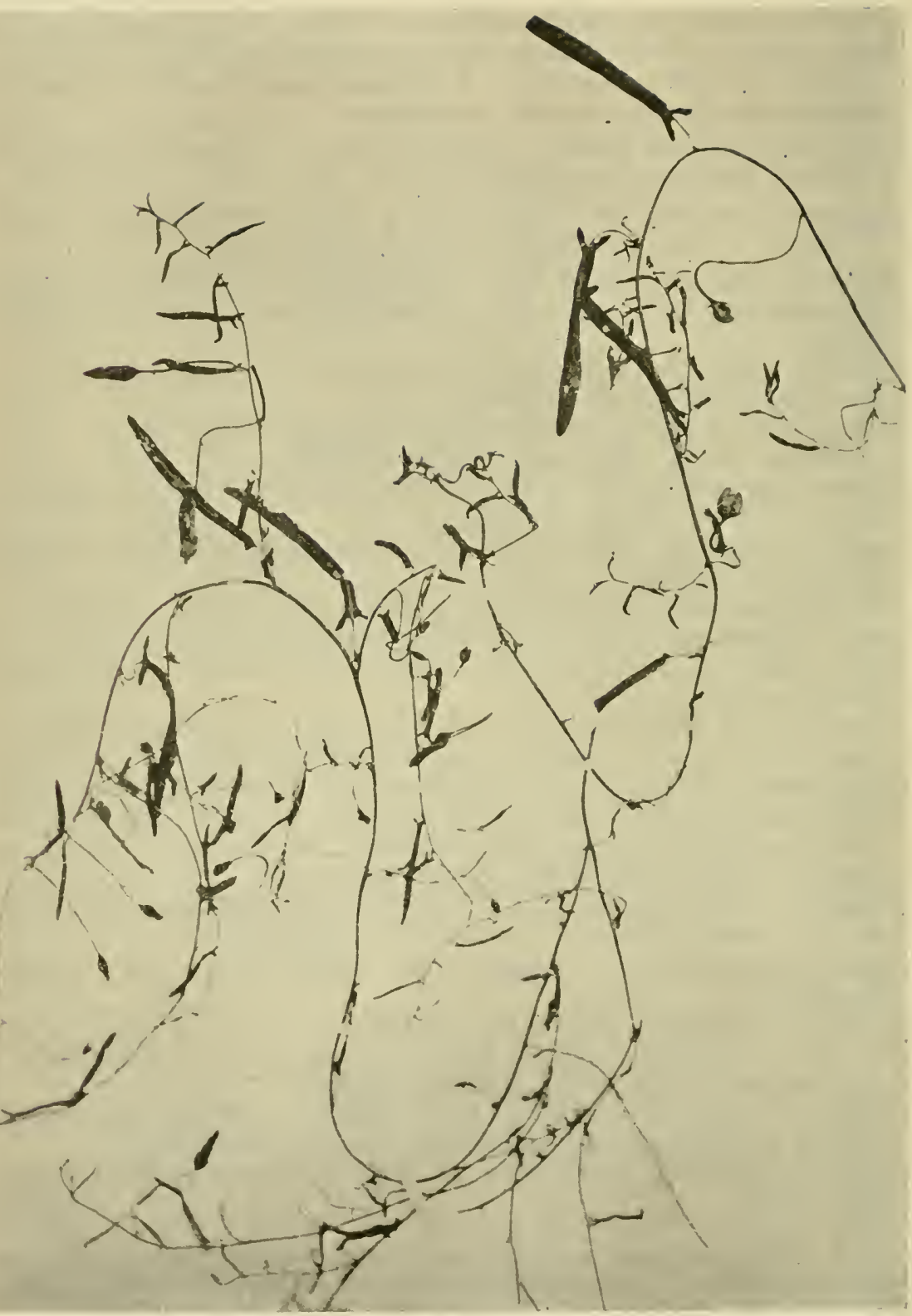

Abb. 3. Convolvulus arvensis L. var. lancifolius Presl. 
Mentha Henvici Borb. var. salina H. B. ${ }^{1}$ ) nova var. Insel Arbe: auf salzhaltigem Boden in Paludo.

Differt a $M$. Hcurici Borb. foliis minoribus, magis pilosis, in marginibus obsolete serratis; ceterum ut in typo.

MI. hirsutu Huds. var. Schleicheri (Opiz), forma urbensis II. B. nova f. Insel Arbe: in den Brackwassergräben am SO-Ende des Loparotales.

Differt a M.hirsuta Huds. foliis magis villosis, fere ovatis, calycibus minus rillosis, ceterum ut in typo.

M. hiruta Huds. var. subpyrifolia II. B. nova var. Insel Arbe: in einem Brackwassergraben am SO-Ende des Loparotales.

Differt a M. pyrifolia H. B. (Belgrad): caules dense villosuli, folia dense pilosa rel subrillosa, breviter petiolata.

11. tomentella Hoffgg. et Link. Insel Arbe: in der Garrigue in der Valle Gožinka.

11. villosa Huds. var. litoralis (Borb.). Insel Arbe: auf salzhaltigem Boden im Paludo.

Asperula laeviguta L. Insel Arbe: im Dundowalde.

A. Staliana Vis. ${ }^{2}$ ). Insel S. Gregorio: in der Formation der Felsflur am NO-Absturze der Insel.

Gulium divaricatum Lam. ${ }^{3}$ ). Insel Arbe: in der steinigen Trift am NOAbsturze des Tignarogebirges.

G. lucidum All. var. Gerardi (Vill.). Inseln Arbe und S. Gregorio: in der steinigen 'Trifi am NO-Absturze beider Inseln.

G. elatum Thuill. Insel Arbe: im Campo Marzio bei der Stadt Arbe (K.); in der Garrigue bei der Kirche S. Élia.

Campanula istriaca Feer. Inseln Arbe, S. Gregorio, Goli und Perricchio: tonangebende Leitpflauze in der Formation der Felsflur der genannten Inseln.

Bellis silvestris Cyr. Insel Arbe: auf anstehendem Flyscbbodell in der Talle S. Pietro bei der Häusergruppe Bernić.

Chrysanthemum platylepis (Borb.) Beck. Insel Arbe: häufig in der Formation der Felsflur am NO-Absturze des Tignarogebirges.

Scnecio vulgaris L. f. carnosus Posp. Ich fand diese Form, die durch ihre stark sukkulenten, bläulich bertiften Blätter sehr auffällig ist, in der Formation des Felsstrandschotters in einzelnen Buchten an der SW. liüste der Iusel Arbe. sowie in der Formation der Strandklippen an den NO-Abstürzen der Inseln Arbe. S. Gregorio und Goli. Bisher aus Istrien bekannt, dürfte die Form, die lediglich als eine Anpassung an starken Salzgehalt des Bodens erscheint, wohl meist übersehen worden sein. Bei einigen Exemplaren wurden auch Strahlblüten beobachtet, was bekanntlich auch beim Typus manchmal rorkommt.

Centaurea dalmatica Kern. Inseln Arbe, S. Gregorio. Goli und Pervicchio: tonangebende Leitpllanze in der Formation der Felstlur der genannten Inseln.

Cichorium pumilum Jacq. *). Insel Arbe: in der steinigen Trift und auf Weideboden auf dem ganzen Tignarogebirge und auf dem Cruna rrh.

1) Alle Nenthen von H. Braun bestimmt.

2) Verglichen m. d. Originalexemplaren des Herb. Visiani durch A. Béguinot.

3) Alle Galien von A. Bégu inot revidiert.

4) Revid. A. Béguinot. 
C. pumilum Jacq. var. divaricatum (Schousb.) Insel Arbe: in der Formation des schotterigen Meeresstrandes in der Valle Matevica.

Nach F'iori, "Flora analitica d'Italia“, III, 386 besitzt $C$. pumilum einen einfachen, stark verkürzten, die var. divaricatum einen ästigen und höheren Stamm. Es sind zwei gut unterscheidbare Formen des C. intylus, die aber geringen systematischen Wert besitzen.

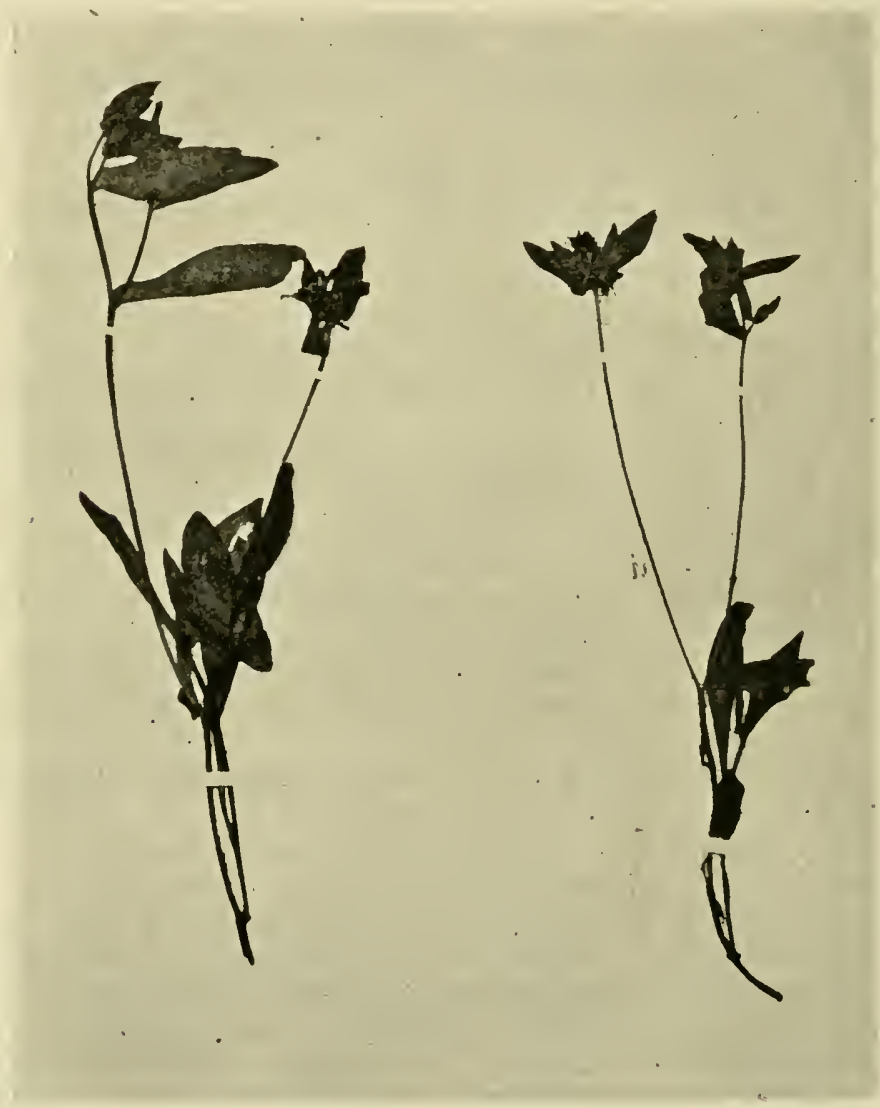

Abb. 4. Picris echioides L. forma.

Picris echioides L. ${ }^{1}$ ) (Abb. 4). Insel Arbe: auf einem Flyschfelsen bei der Kirche S. Elia. Die sonst normal entwickelte Pflanze zeigt eigentümlich ausgebildete äußere Hüllblätter. Sie sind etwas größer als beim Typus und auch an der Innenseite behaart (mit einfachen borstigen Haaren), wodurch sie sich im Ausseben den Stengelblättern nähern. Wir baben jedoch von der Aufstellung einer Form, bzw. Varietät vorderhand noch abgesehen, da ich nur zwei Exemplare fand

1) Det. Eberstaller et Morton. 
und außerdem an eine monströse Bildung, bzw. an die Einwirkung eines Insektes gedacht werden konnte.

Scórzonera austriaca Willd. f. stenophylla Beck. Insel Arbe: in der Formation der Strandklippen am Cap Fronte.

Reichardia picroides (L.) Roth var. maritima Fiori. Inseln Arbe, S. Gregorio und Goli : tonangebende Leitpflanze in der Formation der Felsflur am NO-Absturze der drei Inseln.

Hieracium Bauhini Schult. ssp. clarisetum Morton et Zahn, nova ssp. $\left.{ }^{1}\right)$. Insel Arbe: im Dundowalde.

Caulis gracilis, ad $75 \mathrm{~cm}$ altus, efloccosus, disperse, apice modice setosus et sparsim glandulosus. Folia glauca, efloccosa, modice subsetosociliata (3-4 mm), glabrescentia, oblongo-lanceolata, obtusa, subspathulata, inferiora etiam in parte superiore modice longiusque setosa, caulina 5, lanceolata, sensim decrescentia, remota, setoso-ciliata. Anthela apice umbellata, densiuscule setosa, modice glandulosa, subefloccosa, setis $2-3 \mathrm{~mm}$ longis apice dilutis. Acladium breve, rami ad 7 tenues subcymosi. Ordines axium 3-4, capitula 20 vel compluria. Pedunculi densiuscule setosi, disperse floccosi, subglandulosi. Involucra obscure virescentia, cylindrico-ovata, densiuscule setulosa, disperse glandulosa, efloccosa. Squamae sublatiusculae, dorso pilorum basi nigra obscurae, margine late et pallide virides. Ligulae luteae. Stolones graciles, elongati, efloccosi, modice setosi, foliis remotis sublongis lanceolatis obsiti.

H. pilosella L. ssp. minuticeps N. P. $\beta$. obscurellum Iorton et Zahn. Insel Arbe: im Capofrontewalde.

Squamis margine virescentibus subefloccosis.

Althenia filiformis Fr. Insel Arbe ${ }^{2}$ ): in einem Tümpel bei den Ruinen von S. Damiano. Da am Material entwickelte weibliche Blüten sowie Früchte fehlen, ist die Bestimmung nicht mit absoluter Sicherheit durchzuführen gewesen. Nach G. Fischer spricht für Althenia die ungemein zarte Form der gelblich-weißen Stengel und der büschelig gehäuften, fadenförmigen, nervenlosen Blätter, sowie ein ठð Blütchen, das im Gegensatz zu Zanichellia am Grunde bescheidet ist.

Scilla autumnalis L. Insel Arbe: Verbreitet und häufig im ganzen Kalk der Insel.

Ruscus hypoglossum L. Insel Arbe: im Capofrontewalde bei der Valle Armata.

Juncus Gerardi Lois. Insel Arbe: in der Formation der Meeressimsen am SO-Ende der Campora.

Luzula Forsteri (Sm.) DC. Insel Arbe: im Capofrontewalde.

Crocus longiflorus Raf. Insel Arbe: in der steinigen Trift bei den Ruinen von S. Damiano (Já vorka).

C. reticulatus Stev. Insel Arbe: in der steinigen Trift auf dem Rücken des Tignarogebirges.

Gladiolus illyricus Koch. Insel Arbe: im Dundo- und Capofrontewalde, in den Waldbeständen restlich des Paludo und in der Muudanje glava.

Scirpus litoralis Schrad. Insel Arbe: sehr vereinzelt in Brackwassergräben und -Tümpeln der ganzen Insel.

1) Die Hieracien von $\mathrm{H} \mathrm{Zahn}$ bestimmt.

2) Revid. G. Fischer. 
Carex distachya Desf. ${ }^{1}$ ). Insel Dolin: in der Garrigue im nördlichen Teile der Insel.

C. pallescens L. ${ }^{1}$ ). Insel Arbe: im Dundowalde.

Tragus racemosus (L.) Ail. ${ }^{2}$ ) Insel Arbe: auf Sandäckern in der Valle S. Pietro und auf den Sanddünen am SO-Ende des Loparotales.

Stipa aristella L. Insel Arbe: in der Garrigue am N-Absturze des Cruna vrh.

Phleum arenarium L. Insel Arbe: dominierend auf den Strandwiesen, vereinzelt auf den Sanddünen am S0-Ende des Loparotales.

Ammophila pallida (Presl) Fritsch var. austrulis (Mab.). Insel Arbe: auf den Sanddünen am SO-Ende des Loparotales.

Sesleria tenuifolia Schrad. Insel Arbe: häufig in der steinigen Trift am NO-Absturze des Tignarogebirges.

S. autumnalis (Scop.) Schultz. Insel Arbe: in der Garrigue in der Valle Gožinka; unter elner Steineichengruppe bei der Kirche S. Elia.

Kneleriı splendens Presl. Inseln S. Gregorio und Goli : in der steinigen Trift im westlichen Teile beider Inseln.

Atropis festucaeformis Richter. Insel Arbe: auf den Salinenböden am $\mathrm{NW}$-Ende des Valle S. Pietro.

F'estuca rubra L. var. vulgaris Gaud. subr. glaucophylla Hack. nora subvar. Insel S. Gregorio: in der Formation der Felstlur am NOAbsturze der Insel.

Differt a subr. typica foliis leviter pruinosis (non tantum glaucescentibus). Valde affinis $F$. rubrae subv. pruinosae Hack. in Rep. Bot. Ex. Cl., 1884, p. 119, sed hujus folia subjuncea, panicula angusta etc.

Vulpia dertonensis (All.) Gola. Insel Arbe: in der steinigen Trift auf dem Rücken des Tignarogebirges.

$V$. fasciculata (Forsk.) Fritsch. Insel Arbe: bestandbildend auf den Strandwiesen und vereinzelt auf den Sanddünen am SO-Ende des Loparotales.

Scrapias lingua L. Insel Arbe: im Dundowalde und auf den Flyschkegeln westlich des Paludo (hier auch K.).

Cephialanthera longifolia (L.) Fritsch. Insel Arbe: im Capofrontewalde.

1) Revid. A. Kneucker.

2) Alle Gräser von E. Hackel bestimmt, bzw. revidiert. 




\section{QK 329. Mew York Botanical Garden Library
Morton, Friedrich/Beitrage zur Kenntnis

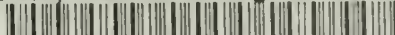

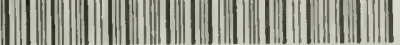 \\ 35185001101821}


